\title{
Experiencias y obstáculos de los psicólogos en el acompañamiento de los procesos de fin de vida
}

\author{
Manuel Fernández-Alcántara ${ }^{1 *}$, M. Paz García-Caro ${ }^{2}$, M. Nieves Pérez-Marfil ${ }^{1}$, Francisco Cruz-Quintana ${ }^{1}$ \\ ${ }^{1}$ Departamento de Personalidad, Evaluación y Tratamiento Psicológico, Facultad de Psicología, Universidad de Granada, España \\ ${ }_{2}^{2}$ Departamento de Enfermería, Facultad de Ciencias de la Salud, Universidad de Granada, España
}

\begin{abstract}
Resumen: Los psicólogos están encargados de realizar la atención emocional a los pacientes que se encuentran en procesos de fin de vida. No se conocen cuáles son las experiencias, los obstáculos y problemas con que se encuentran al intervenir en estos casos, siendo éste el principal objetivo de la investigación. Para ello se realizó un estudio cualitativo de tipo fenomenológico utilizando entrevistas en profundidad, que se analizaron con el software Atlas.ti siguiendo una codificación abierta. La muestra estuvo compuesta por 15 psicólogos que desempeñaban su labor en cuidados paliativos, otras unidades hospitalarias (oncología, salud mental, urgencias y atención temprana) o intervenían de manera privada en la provincia de Granada (España). Los resultados muestran la variedad de experiencias en esta intervención, así como la existencia de diferentes obstáculos que dependen del momento de intervención, de las características propias del paciente o la familia, las emociones del psicólogo, o la organización sanitaria. Se ponen de relevancia cómo los propios sentimientos de los psicólogos pueden influir en su intervención, y se propone la necesidad de una formación para superar los diferentes obstáculos que plantea la intervención en el final de vida.

Palabras Clave: psicología; estudio cualitativo; profesionales de la salud; enfermedad terminal; cuidados paliativos.
\end{abstract}

\section{Introducción}

Según el American National Institute Health el proceso de fin de vida está formado por una serie de componentes (Ostgathe y Voltz, 2010). Estaríamos ante la presencia de personas que padecen una enfermedad crónica, síntomas o alteraciones funcionales que persisten, y que pueden ser fluctuantes, o ante personas con una edad avanzada asociada o no a enfermedad. Los síntomas, derivados de una enfermedad irreversible, deben requerir atención profesional o familiar, y pueden llevar a la muerte (Ostgathe y Voltz, 2010).

En los últimos años, gracias al aumento de la conciencia y demanda social y al auge y desarrollo de los Cuidados $\mathrm{Pa}$ liativos, que dan una atención multidisciplinar a los diferentes aspectos de la persona en los procesos de final de vida (Bayés, 2001; Pastrana, Jünger, Ostgathe, Elsner y Radbruch, 2008), están surgiendo debates sobre la calidad de la muerte y la necesidad de un mayor cuidado en la etapa final de la vida. En España, la atención paliativa de las personas no está aún generalizada, lo que conlleva que, por lo general, se muere mal (García-Caro et al., 2008a; 2008b). Son muy relevantes los obstáculos con que se encuentran los profesionales sanitarios a la hora de dar el diagnóstico de terminalidad, debido a la gran dificultad emocional que supone para ellos (García-Caro et al., 2010; Mialdea, Sanz y Sanz, 2009).

* Dirección para correspondencia [Correspondence address]: Manuel Fernández-Alcántara. Facultad de Psicología. Campus de Cartuja s/n 18071 Granada (España). E-mail: manufer@,correo.ugr.es
Title: Experiences and obstacles of psychologists in the accompaniment of end-of-life processes.

Abstract: Psychologists are responsible for the emotional care to patients who are in end-of-life processes. Little is known about the experiences, the obstacles and problems they face when addressing such complex situations, so the main objective of this research is to understand and explore what they are. A qualitative phenomenological design was performed, using semi structured interviews, which were analyzed with the software Atlas.ti following an open coding. The sample consisted of 15 psychologists who perform their work in palliative care, other hospital units (oncology, mental health, emergency and early intervention) or privately involved in the province of Granada (Spain). The results show how psychologists contact with patients in end-of-life processes, as well as the variety of both positive and negative experiences in their speech. It is shown the existence of various obstacles that have to do with the timing of intervention, characteristics of the patient or family, with the emotions of the psychologist, as well as the health organization. This study shows how the feelings of the psychologists can influence their intervention, which raises the need for a specific training where they can learn different strategies to overcome the obstacles posed by intervention at the end-of-life.

Keywords: psychology; qualitative study; health professionals; terminal illness; palliative care.

El área psicológica y el cuidado de los aspectos emocionales del paciente y de la familia son fundamentales al intervenir en el final de vida (Barreto y Bayés, 1990). Los psicólogos son parte esencial de esta intervención (Werth Jr, Gordon y Johnson Jr, 2002) y la forma en que ellos afrontan $\mathrm{y}$ atienden los procesos de fin de vida es diferente a la de otros profesionales sanitarios. La intervención se produce en diversos ámbitos como las unidades de cuidados paliativos, áreas hospitalarias como salud mental, emergencias o atención temprana (García-Fernández y et al., 2008; RomaníCosta, Expósito-López, Rodriguez-Carballeira y AlmagroMena, 2010), así como en los gabinetes psicológicos donde acuden casos de terminalidad o duelo.

Si bien se conocen y están definidos los roles y las labores del psicólogo en cuidados paliativos (Lacasta, RocafortGil, Blanco-Toro, Limonero-García y Gómez-Batiste, 2008; López-Ríos y Ortega-Ruiz, 2005), se desconocen cuáles son las experiencias, los problemas y obstáculos que éstos encuentran al desarrollar su labor. Se ha estudiado ampliamente la problemática e impedimentos con los que se encuentran los profesionales médico-sanitarios, asociados entre otros, a dificultades para delimitar el diagnóstico de terminalidad, la comunicación de malas noticias, el papel crucial de la familia, o los problemas emocionales propios al trabajar en el fin de vida (García-Caro et al., 2010; Groot, VernooijDassen, Crul y Grol, 2005; Mialdea et al., 2009; Schmidt RíoValle et al., 2009), pero no se encontraron trabajos que hayan investigado estas problemáticas en los psicólogos. 
El objetivo de esta investigación es conocer las experiencias y los obstáculos de los psicólogos en sus intervenciones en los procesos de fin de vida. Este objetivo se concreta en las siguientes preguntas de investigación:

1. Determinar qué papeles desempeñan los psicólogos entrevistados frente a situaciones de muerte y dolor, y cómo entran en contacto con los procesos de fin de vida.

2. Conocer las experiencias, tanto positivas como negativas, cuando trabajan en la fase final de la vida.

3. Identificar los obstáculos, problemas o impedimentos en la práctica clínica a nivel general y específico.

\section{Método}

Se diseño un estudio cualitativo de corte fenomenológico fundamentado en la Grounded Theory (Glaser y Strauss, 1967) cuyo universo fueron profesionales psicólogos que trabajasen o interviniesen en el final de vida en la provincia de Granada (España). Se seleccionaron psicólogos de unidades de cuidados paliativos, de áreas hospitalarias y servicios especiales (atención temprana, salud mental, oncología y urgencias), así como aquellos que ejercían la práctica privada en consultas u organizaciones, interviniendo en duelo y enfermedades terminales. La muestra estuvo constituida por 15 profesionales (Tabla 1) seleccionados mediante muestreo intencional (Flick, 2004), con una edad media de 36,33 años $(D T=8.7)$, siendo un $26.7 \%$ hombres $(N=4)$ y un $73.3 \%$ mujeres $(N=11)$.

Los criterios de homogeneidad fueron ser psicólogo, estar en ejercicio activo, y tener relación, en su práctica profesional, con la intervención en procesos de fin de vida. Los criterios de heterogeneidad han sido el sexo, el lugar del ejercicio de la profesión, y el carácter público o privado del ejercicio profesional.

Los criterios de inclusión fueron que los psicólogos tuvieran experiencia profesional en los procesos de fin de vida y que aceptasen participar en el estudio. Los criterios de exclusión fueron no trabajar actualmente en la provincia de Granada (España), y no aceptar participar en la investigación.

Los datos se recogieron a través de entrevistas semiestructuradas, cuyo guión fue elaborado ad hoc a partir de una Tabla 2. Principales familias de códigos utilizados en la investigación

\begin{tabular}{llll}
\hline Tabla 2. Principales familias de códigos utilizados en la investigación & \\
\hline & Dificultades Generales & Dificultades con los Pacientes & $\begin{array}{l}\text { Dificultades } \\
\text { con las Familias }\end{array}$ \\
& Organizacionales: & & $\begin{array}{l}\text { Dificultades } \\
\text { con el Equipo Sanitario }\end{array}$ \\
& - Tiempo & & \\
& - Organizativos & Experiencias Positivas & Experiencias Positivas \\
Sentimiento Positivo Trabajo & - Rol & Problemas & Problemas \\
Sentimiento Negativo Trabajo & Personales: & Características Positivas & Papel Familia \\
Forma de Intervención & - Emocionales & Intervención en Niños & Dificultades Inicio \\
& - Comunicación & Intervención en Duelo & \\
& Derivación & &
\end{tabular}

revisión bibliográfica, que permitió establecer los principales ejes del guión de temas y preguntas (ver Anexo). Se realizaron en los meses de Abril a Julio de 2011.

\begin{tabular}{llll}
\multicolumn{3}{l}{ Tabla 1. Características generales de la muestra. } \\
$\begin{array}{l}\text { Grupo de } \\
\text { Psicólogos }\end{array}$ & Número & Sexo & Profesión \\
\hline $\begin{array}{l}\text { Cuidados } \\
\text { Paliativos }\end{array}$ & 5 & 4 mujeres & $\begin{array}{l}\text { Unidades de cuidados paliativos (I3, } \\
\text { I5, I13 e I15) }\end{array}$ \\
\cline { 2 - 3 } $\begin{array}{l}\text { Áreas } \\
\text { Hospitalarias }\end{array}$ & 6 & 4 mujeres & $\begin{array}{l}\text { Atención temprana (I2 e I9), Salud } \\
\text { Mental (I10) y Oncología (I 12) }\end{array}$ \\
\cline { 2 - 4 } & 2 hombres & $\begin{array}{l}\text { Emergencias ( I7) y Atención tem- } \\
\text { prana (I4) }\end{array}$ \\
\hline $\begin{array}{l}\text { Práctica } \\
\text { Privada }\end{array}$ & 4 & 3 mujeres & $\begin{array}{l}\text { Asociación (I6 e I11) y Gabinete (I1) } \\
\text { Gabinete (I8) }\end{array}$ \\
\hline
\end{tabular}

Antes de la realización de la entrevista se les entregaba el consentimiento informado y se abría un espacio para dudas con respecto al estudio. La entrevista fue llevada a cabo por el mismo investigador, procurando que las condiciones fuesen similares. En caso de que existiera alguna diferencia o particularidad se señalaba en la hoja de incidencias. Las entrevistas se desarrollaron en un único encuentro con cada entrevistado, con una duración que osciló entre 46 y 110 minutos. Una vez realizadas, fueron transcritas mediante el software F4.

Para el proceso e interpretación de los datos cualitativos se ha utilizado el software Atlas.ti en su versión 5.0 (Lewis, 2004; Scientific Software Development, 2011), utilizando una estrategia de codificación abierta (Strauss y Corbin, 1998). El programa Atlas.ti utiliza un sistema de análisis basado en la codificación del texto. En primer lugar se asignan los documentos primarios (entrevistas transcritas) a la base de datos o unidad hermenéutica $(\mathrm{HU})$ creada, para, en segundo lugar, generar las citas o segmentaciones del texto significativas y codificarlas (asignarles diferentes códigos). Por último, se establecen relaciones (entre códigos o entre códigos y citas), y se crean redes (network) donde se muestra la teoría explicativa a partir del discurso de los participantes (Muhr,1991).

Para la generación de códigos se han seguido dos pasos diferentes. Por un lado, se ha trabajado con códigos que se han derivado del marco conceptual previo, así como de los objetivos y las diferentes secciones de la entrevista. Por otro, durante el análisis y lectura de las entrevistas se han ido formando nuevos códigos, denominados emergentes (Tabla 2). 
Para elaborar las redes de relaciones, mediante la herramienta de búsqueda de este programa, es necesario desarrollar un sistema de segmentación del texto en relación a unos códigos genéricos, que han de estar asociados a todas las citas, y unos códigos específicos relativos a la temática concreta del texto. Los códigos genéricos que se han utilizado han sido la edad, el sexo, el nivel de estudios, la profesión/lugar de trabajo, la situación laboral y la orientación teóricopráctica. Los códigos específicos son los que se han agrupado en familias de códigos y se muestran organizados en relación con las dimensiones del análisis o categorías finales.

Para mejorar la confiabilidad del estudio se controlaron la génesis de los datos, así como la transcripción de las entrevistas. En el primer caso, se ha diferenciado de manera clara entre las citas textuales procedentes de los participantes y las interpretaciones o resúmenes realizados por los investigadores. En el segundo, las entrevistas han sido transcritas por el mismo investigador, manteniendo unos criterios homogéneos a lo largo de las mismas. Por último, el proceso de análisis y elaboración de los resultados ha estado sometido a la triangulación entre investigadores para garantizar la credibilidad de los mismos.

\section{Resultados}

Papeles que desempeñan los psicólogos entrevistados frente a situaciones de muerte y dolor, y cómo entran en contacto con los procesos de fin de vida

La intervención en los procesos de fin de vida supone una gran tensión emocional, siendo especialmente relevantes los primeros momentos, en los que el psicólogo contacta con los pacientes y las familias. Las intervenciones que se realizan en cuidados paliativos tienen la particularidad de que intentan no abrir nada que pueda ser problemático, es decir, tratan de disminuir la ansiedad en el paciente y en la familia sin profundizar demasiado. Por lo general, la intervención en el fin de vida es más complicada cuando trabajan en el hospital. En ocasiones deben intervenir en situaciones de urgencia, lo que exige actuar sin la información adecuada y sin una preparación previa sobre el caso concreto. Además, determinadas áreas hospitalarias, como puede ser urgencias, dificultan que se le dedique el tiempo suficiente a la relación terapéutica (Figura 1).

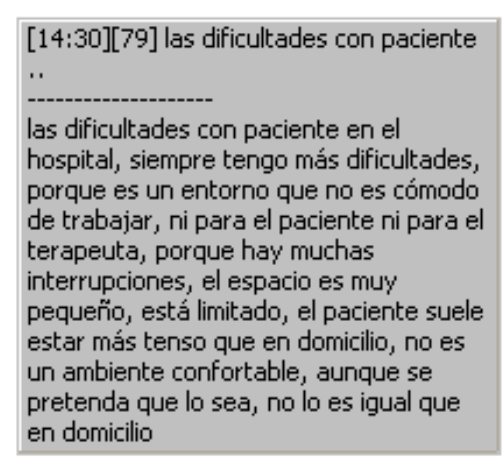

Profesión 2 - Hospital

En una área hospitalaria que no es CP

[2:17][148] somos como el psicólogo al que..

somos como el psicólogo al que se recurre en un momento de tensión, pero que no está en el proceso. Entonces lo que es importante es que pueda estar en el proceso. Porque las familias se sienten también que es que llaman al psicólogo porque ellos no están dando la talla, y eso es un problema

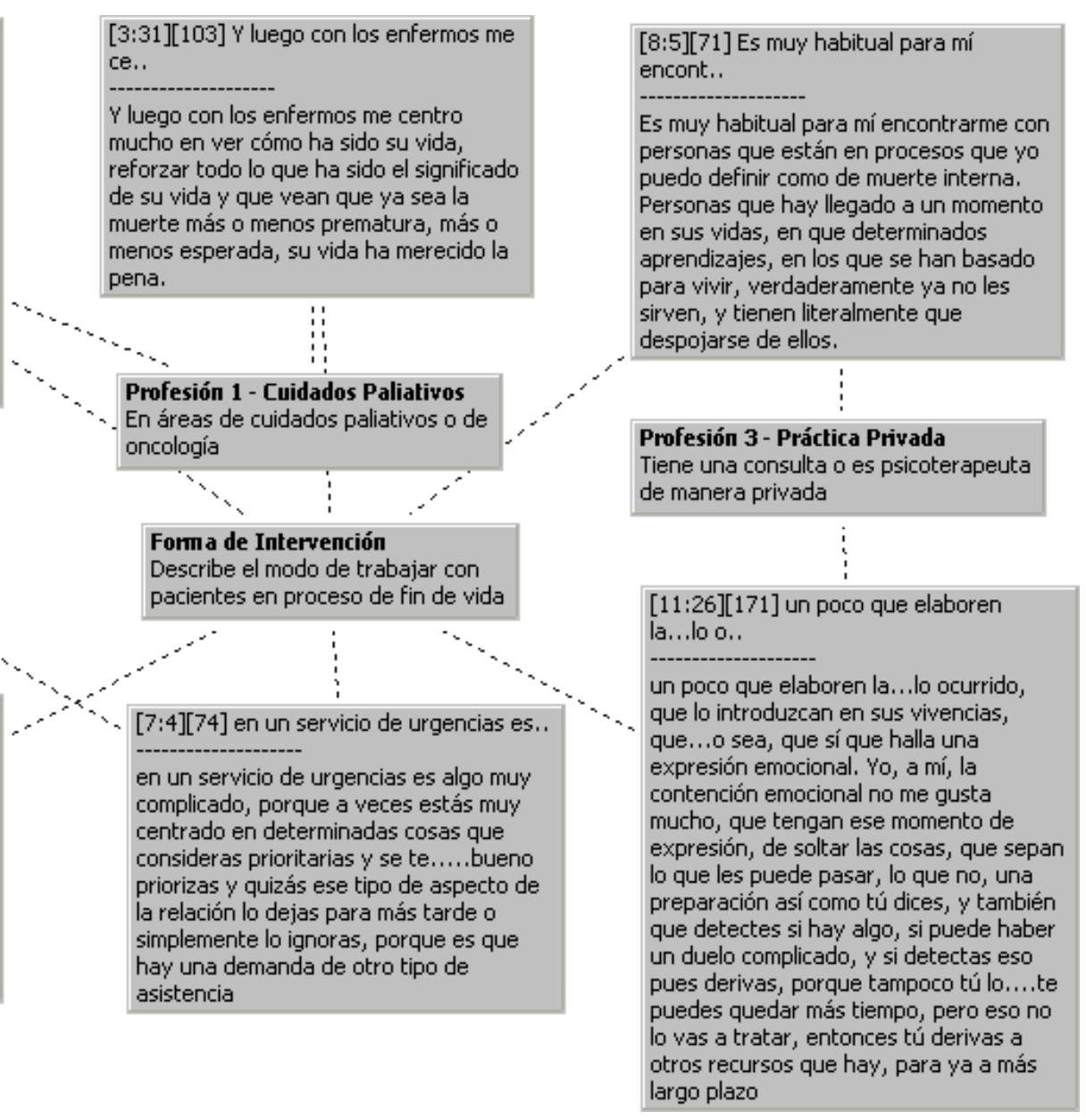

Figura 1. Códigos y citas sobre las características de la intervención en el fin de vida. 
El tipo de pacientes sobre los que intervienen en el ámbito del fin de vida está mediado por el lugar de trabajo. Los psicólogos que trabajan en cuidados paliativos intervienen principalmente con pacientes oncológicos, sus familias y con el equipo sanitario. Aquellos que trabajan en áreas hospitalarias centran su intervención en niños o en sus familias, así como en pacientes con intentos de suicidio. Finalmente, los que trabajan de manera privada, además de personas en duelo y en procesos oncológicos, mencionan los duelos por pérdidas relacionales (ej. romper con la pareja), interpersonales (ej. envejecer), y personas con un diagnóstico de terminalidad que acuden a tratar alguna cuestión que consideran que está pendiente en su vida.

La forma en la que se establece el primer contacto con los pacientes en proceso de fin de vida, es también diferente. Los psicólogos que trabajan en el hospital reciben derivaciones, algo que no ocurre con tanta frecuencia en las unidades de cuidados paliativos ni en los gabinetes privados, donde son los propios psicólogos quienes realizan un primer cribado.
Experiencias de los psicólogos cuando trabajan en la fase final de la vida

Lo que más gratifica y satisface a estos psicólogos es poder ayudar a otros en los últimos momentos de vida, así como la gratitud que muestran los pacientes y/o las familias durante y tras las intervenciones. También destacan poder ver cómo evoluciona el paciente, el agradecimiento y la participación de las familias, así como el apoyo emocional que puede proporcionar el equipo sanitario. Las experiencias negativas que tienen en su trabajo son más variadas y parecen depender de características personales. Lo que aparece con más frecuencia son las sensaciones de cansancio, tanto mental como físico, relacionadas con la gran carga asistencial, así como el malestar e impotencia en ciertas situaciones del fin de vida (Figura 2).

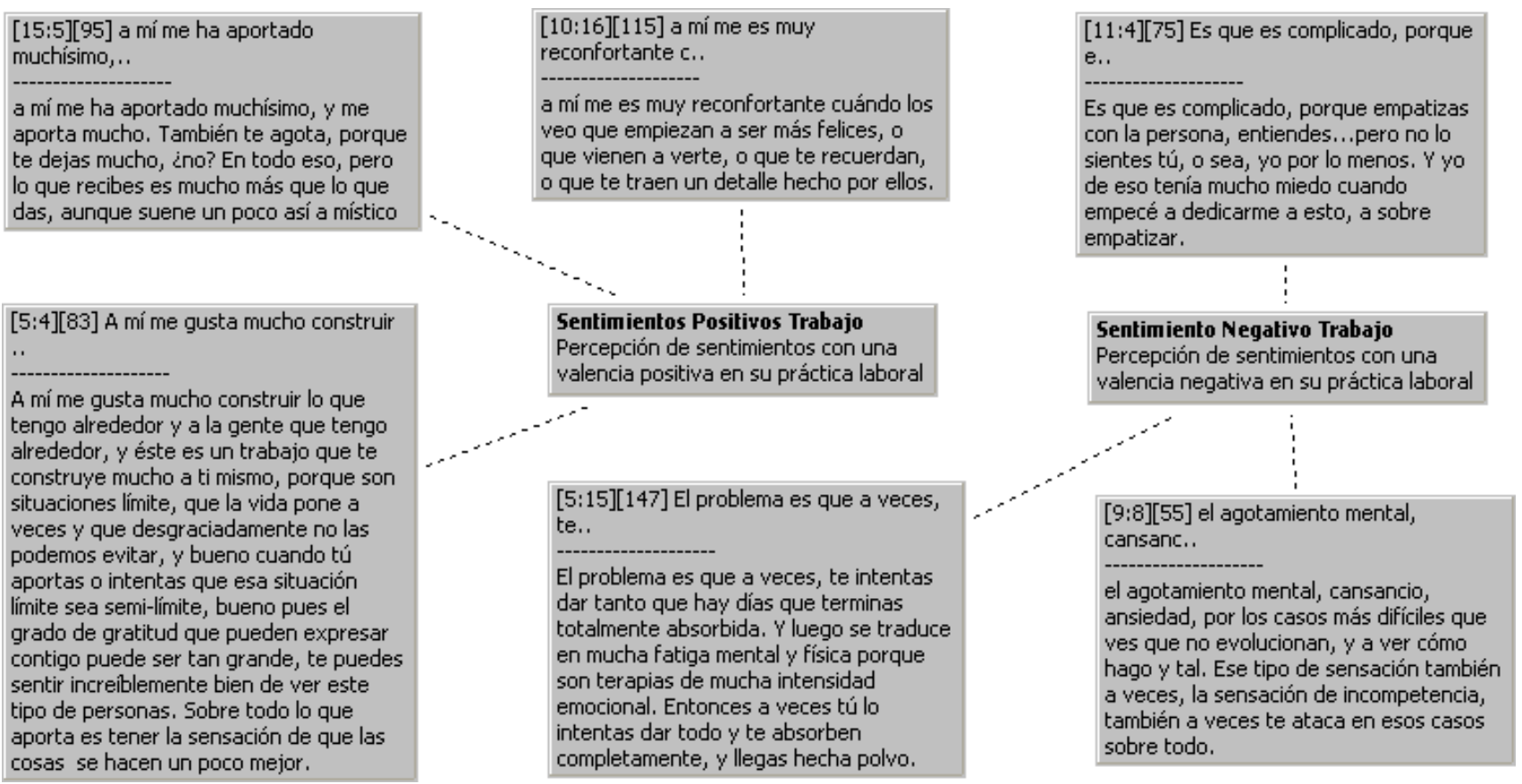

Figura 2. Códigos y citas sobre las experiencias de los psicólogos al trabajar en el fin de vida.

Obstáculos, problemas e impedimentos en la práctica clínica de los psicólogos a nivel general y específico

\section{Obstáculos organizacionales}

Los psicólogos que trabajan en hospital han señalado más problemas en esta área. Tienen que ver con el estado en que se encuentra el lugar de trabajo, así como la falta de privacidad para hablar con los pacientes. Los psicólogos de cuidados paliativos señalan que a su unidad se la tiene muy poco en cuenta, y que la disposición del hospital dificulta la intervención.

Con respecto al tiempo, los psicólogos entrevistados no han encontrado que sea un obstáculo, siempre y cuando fuesen ellos mismos los que organizaran ese tiempo, en la mayoría de las ocasiones en contra de la normativa vigente. En los demás casos, en los que el psicólogo se ajusta al tiempo que tiene estipulado, sí lo percibe como un obstáculo, pues- 
to que le impide realizar lo que considera son sus intervenciones y funciones (Figura 3).

\section{Obstáculos personales}

Los principales obstáculos señalados son la dificultad para manejar las emociones, así como las situaciones en las que el propio psicólogo está pasando por un proceso similar, o lo tiene todavía muy reciente. Con respecto a la comunicación, se ha encontrado que en muchos casos el personal médico-sanitario delega algunas de sus funciones en el psicólogo, bajo la consigna de que ellos se encuentran mejor formados. Así, el psicólogo se convierte en el encargado de dar malas noticias, o de informar sobre aspectos como la quimioterapia y sus efectos negativos. También se crean situaciones muy complicadas en los casos en los que nadie se responsabiliza de dar la información (Figura 3).

\section{Obstáculos relacionados con la derivación del paciente}

Por lo que se refiere a las derivaciones, por norma general, el personal médico no deriva al psicólogo en el tiempo adecuado. Así, cuando ve por primera vez a muchos de sus pacientes éstos se encuentran en un estado de extrema gravedad, lo que dificulta que realicen una intervención adecuada. Algo parecido ocurre cuando se trata de las familias, donde las intervenciones son también muy breves, debido al momento en que llegan al psicólogo. El tiempo de derivación no es considerado como un obstáculo en los casos en los que el psicólogo es el primero que recibe a los pacientes, y puede hacer un screening inicial. Los psicólogos que trabajan en gabinete privado tampoco lo consideran un obstáculo, puesto que son los pacientes los que acuden a ellos (Figura $3)$.

\begin{tabular}{l}
\hline [10:21][131] a los médicos les daba \\
mucho m.. \\
a los médicos les daba mucho miedo de \\
poder hablar de eso, porque muchas \\
veces nos llaman para dar las malas \\
noticias, porque ellos no soportan dar la \\
mala noticia, no porque ellos no sepan, \\
sino porque internamente ellos no lo \\
soportan. Entonces llaman al \\
psicólogo....como podemos estar \\
habituados a las malas noticias. \\
Entonces, el obstáculo era....el que \\
siempre tee encuentras dentro de un \\
modelo médico \\
\hline \\
$\begin{array}{l}\text { Problem as Comunicación } \\
\text { Señala que durante su práctica tiene } \\
\text { problemas relacionados con la } \\
\text { comunicación }\end{array}$ \\
\hline
\end{tabular}

\section{Problemas Emocionales \\ Durante su práctica tiene problemas relacionados con el manejo de las emociones}

(1)

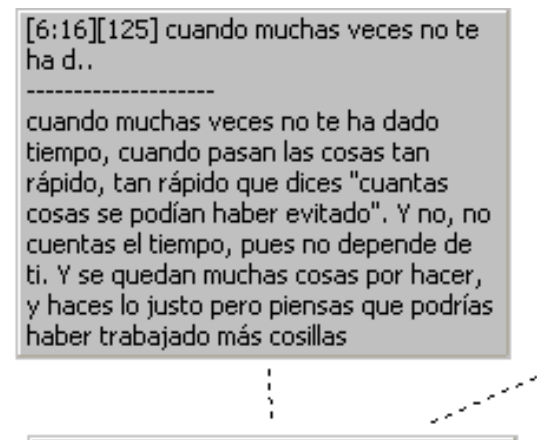

Señala que el tiempo es una variable que supone un obstáculo para su práctica

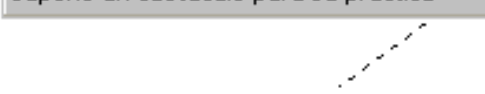

Encuentra obstáculos que tienen que ver con la organización de su lugar de trabajo

[3:32][135] un poco de ansiedad tengo en e..

un poco de ansiedad tengo en esas situaciones, porque no sé lo que me voy a encontrar, cómo de dificultoso me va a resultar, sobre todo esa primera conversación dónde yo tengo que conseguir, o mi objetivo es conseguir la confianza del paciente en mí, para que luego con alguna visita más pues pueda conseguir ahondar en algo que no es fácil ahondar. Normalmente la ansiedad la llevo, pero no me veo yo con, por lo menos no tengo yo la sensación de que me falten habilidades

\section{Problemas Tiempo}

Problemas Organizativos

\begin{tabular}{|c|c|}
\hline $\begin{array}{l}{[6: 16][125] \text { cuando muchas veces no te }} \\
\text { ha d.. }\end{array}$ & $\begin{array}{l}{[3: 6][131] \text { el funcionamiento del }} \\
\text { hospital.. }\end{array}$ \\
\hline $\begin{array}{l}\text { cuando muchas veces no te ha dado } \\
\text { tiempo, cuando pasan las cosas tan } \\
\text { rápido, tan rápido que dices "cuantas } \\
\text { cosas se podian haber evitado". Y no, no } \\
\text { cuentas el tiempo, pues no depende de } \\
\text { ti. Y se quedan muchas cosas por hacer, } \\
\text { y haces lo justo pero piensas que podrias } \\
\text { haber trabajado más cosillas }\end{array}$ & $\begin{array}{l}\text { el funcionamiento del hospital en sí } \\
\text { mismo, que lleva un engranaje de mucha } \\
\text { gente trabajando con la misma persona y } \\
\text { ahí entran auxiliares, enfermeros, } \\
\text { médicos, el fisio, todo el mundo y muchas } \\
\text { veces cuándo tú te buscas el momento, } \\
\text { pues no es el momento adecuado. Y } \\
\text { cuando te lo piden a tí, pues tú en ese }\end{array}$ \\
\hline i & haciendo otra \\
\hline $\begin{array}{l}\text { Problem as Tiempo } \\
\text { Señala que el tiempo es una variable que } \\
\text { supone un obstáculo para su práctica }\end{array}$ & \\
\hline & $\begin{array}{l}{[2: 15][128] \text { Te envían, tampoco sabes }} \\
\text { muy b.. }\end{array}$ \\
\hline $\begin{array}{l}\text { Problem as Organizativos } \\
\text { Encuentra obstáculos que tienen que ver } \\
\text { con la organización de su lugar de trabajo }\end{array}$ & $\begin{array}{l}\text { Te envían, tampoco sabes muy bien, a lo } \\
\text { mejor tienen cuestiones, o te dicen que } \\
\text { está de una manera el niño y luego, por }\end{array}$ \\
\hline $\begin{array}{l}\text { Problem as Derivación } \\
\text { Durante su práctica encuentra } \\
\text { obstáculos relacionados con el momento } \\
\text { de derivación de los pacientes }\end{array}$ & $\begin{array}{l}\text { situación no era tan grave, o era más o } \\
\text { menos graves. Entonces en esos } \\
\text { sentidos a mí me resulta una experiencia } \\
\text { mala }\end{array}$ \\
\hline$[13: 11][67]$ a veces, no un malestar, & $\begin{array}{l}{[15: 4][179] \text { hay situaciones en que las }} \\
\text { der.. }\end{array}$ \\
\hline $\begin{array}{l}\text { sino .. } \\
\text { a veces, no un malestar, sino que son } \\
\text { situaciones lo mismo muy fuertes, alguna } \\
\text { persona joven, o que tú veas y que está } \\
\text { a punto de.... que ha venido aquí para } \\
\text { que se lleve fármacos para la sedación, y } \\
\text { tú sabes que ya, vamos, que lo estás } \\
\text { viendo y ya no....Entonces eso te } \\
\text { provoca un poco de malestar }\end{array}$ & $\begin{array}{l}\text { hay situaciones en que las derivaciones } \\
\text { se hacen muy tarde, a nivel ya } \\
\text { psicológico, de decir "joder, si es que } \\
\text { esto si lo hubiéramos cogido antes, nos } \\
\text { habría dado tiempo a trabajar muchas } \\
\text { cosas", y eso lo notas, o sea el trabajo } \\
\text { preventivo a nivel psicológico, a nivel de } \\
\text { elaboración, a nivel de.....eso lo notas, } \\
\text { cuándo lo coges a tiempo, y tienes } \\
\text { tiempo de hacer eso es magnifico. }\end{array}$ \\
\hline
\end{tabular}

Figura 3. Códigos y citas sobre los obstáculos que encuentran los psicólogos en el fin de vida I. 


\section{Obstáculos al trabajar con el paciente, la familia y el equipo sa- nitario}

Los obstáculos percibidos en la intervención con el paciente son muy heterogéneos, y parecen depender mucho del lugar de trabajo. Para los psicólogos que trabajan en paliativos el principal problema se produce al identificarse con el paciente, ya sea porque tienen la misma edad, o porque comparten con ellos alguna característica. Son complicadas las situaciones en que el paciente no quiere saber nada de su enfermedad, los intentos de suicidio, y los casos en que el pronóstico no está claro y es cambiante.
Los principales obstáculos que se encuentran al trabajar con las familias son la conspiración de silencio, la no aceptación del diagnóstico y la existencia de psicopatología previa en la familia.

Finalmente, las dificultades con el equipo sanitario giran alrededor de la disparidad de criterios a la hora de atender a un determinado paciente, la falta de coordinación entre compañeros, los sentimientos negativos cuando creen que un compañero está actuando de manera inadecuada (sea ésta de fin de vida, o no), y la crítica entre ellos. En ocasiones, las expectativas que pone el equipo sobre el psicólogo hacen que a éste le cueste mostrarse vulnerable o con dificultades emocionales al intervenir en el fin de vida (Figura 4).

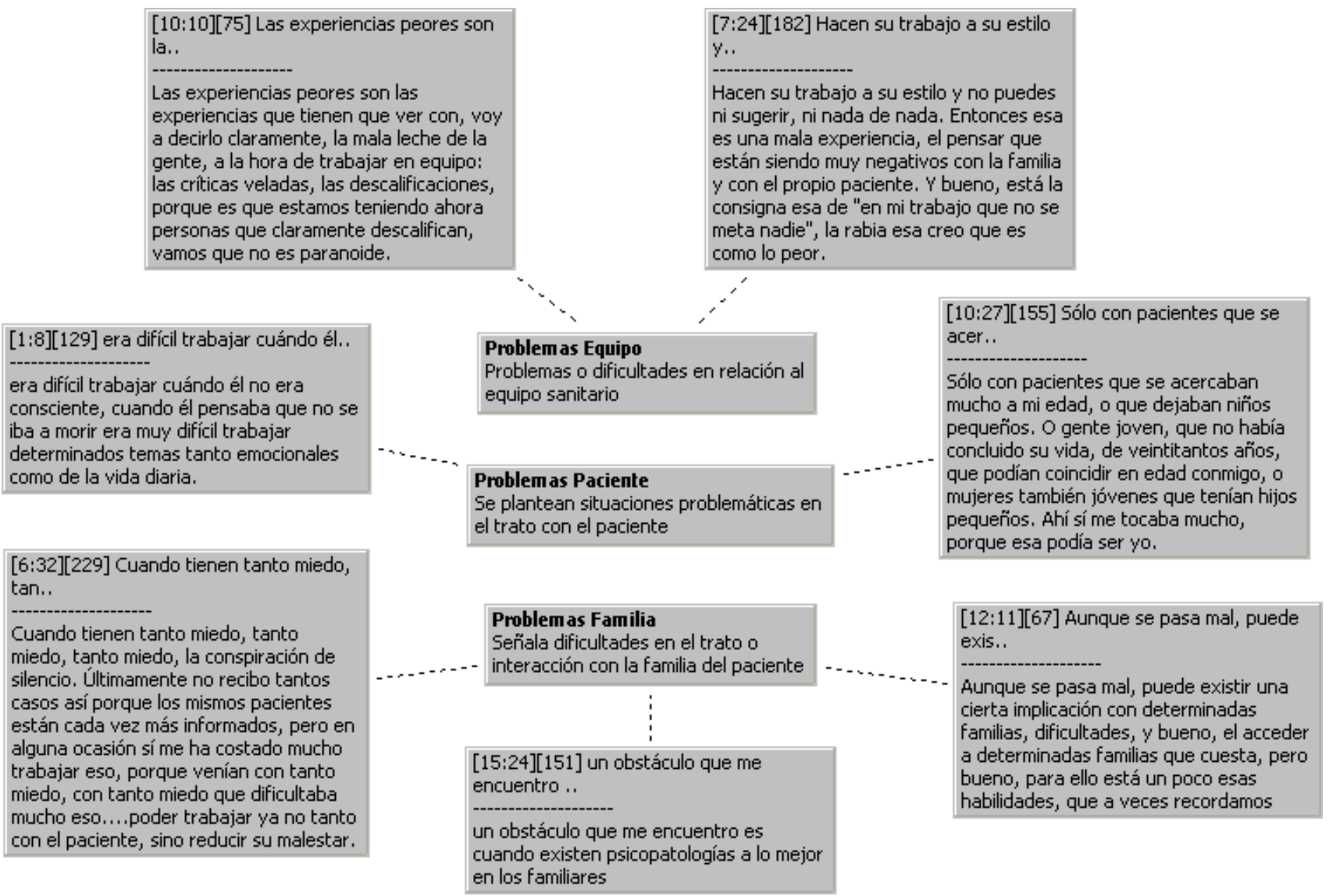

Figura 4. Códigos y citas sobre los obstáculos que encuentran los psicólogos en el fin de vida II.

\section{Discusión}

El estudio muestra cuáles son algunos de los obstáculos y problemáticas que se encuentran los psicólogos al intervenir en el fin de vida. Los papeles o roles que desempeñan coinciden con aquellos incluidos en otros estudios (Haley, Larson, Kasl-Godley, Neimeyer, y Kwilosz, 2003; Lacasta et al., 2008), aunque éstos no realizan intervenciones preventivas (Haley et al., 2003). Los pacientes que disfrutan de aten- ción en el fin de vida son principalmente aquellos diagnosticados por procesos oncológicos, lo que deja a una gran población desatendida (Gardiner, Cobb, Gott e Ingleton, 2011). Este resultado no coincide con otros estudios realizados en España, que encuentran que éstos eran el 50\% de la población atendida (Lacasta et al., 2008). Aquellos que trabajan en áreas hospitalarias han señalado, como un gran problema en el fin de vida, la inexistencia de protocolos adecuados para la atención por parte del sistema sanitario, lo que da 
como resultado que muchas de las defunciones que se producen en el hospital sean precedidas por periodos de agonía, que podrían evitarse (Romaní Costa et al., 2010; Gardiner et al., 2011).

Trabajar en el fin de vida proporciona a todos los psicólogos un gran número de vivencias positivas, que superan con creces a las negativas. Estos resultados pueden deberse a lo reforzante que resulta trabajar en este ámbito, puesto que, aunque la intervención es dura, los pacientes y las familias suelen agradecer mucho la labor realizada. La literatura confirma estos hallazgos, especialmente en aquellos sanitarios que trabajan en cuidados paliativos, señalando que si bien es un trabajo complejo y difícil, también es gratificante (Groot et al., 2005). Recientes estudios han señalado que el personal de dichas unidades suele disfrutar de una mayor satisfacción laboral (Pierce et al., 2007) y menor burnout (CasadoMoragón, Muñoz-Durán, Ortega-González, y CastellanosAsenjo, 2005). Por el contrario, los psicólogos que trabajan en otras áreas hospitalarias no expresan estar tan satisfechos con su trabajo, quizás por la gran presión asistencial, o por el poco tiempo de que disponen para intervenir, obstáculos que tienen que ver con la forma en que se encuentra organizado el sistema hospitalario.

Los hallazgos encontrados en relación a la escasa derivación coinciden con estudios previos, poniendo de manifiesto que las situaciones en las que se demanda un psicólogo en el fin de vida son aquellas dónde la condición del paciente es ya muy compleja y desesperada (Romero, Álvarez, Bayés y Schröder, 2001).

Los obstáculos emocionales tienen que ver con dificultades al manejar los sentimientos de impotencia, sufrimiento y ansiedad que sienten al enfrentarse al paciente que se encuentra en un proceso de fin vida. Hay ocasiones en las que el psicólogo puede identificarse con pacientes que se parezcan a él físicamente o que se encuentren en una situación vital similar, lo que dificulta la intervención si el psicólogo empatiza demasiado. En este sentido se hace necesaria una formación en el fin de vida que incluya aspectos prácticos y vivenciales que doten al psicólogo de recursos para poder afrontar éste tipo de situaciones complejas (FernándezFernández, 2003).

La literatura pone de relevancia que en España el diagnóstico de enfermedad terminal y la comunicación de las malas noticias traen consigo muchas complicaciones a nivel emocional, siendo considerado como uno de los peores aspectos del trabajo sanitario (García-Caro et al., 2010; Schmidt Río-Valle et al., 2009). En el caso de los psicólogos no se han encontrado grandes obstáculos en la comunicación, al contrario que en otros profesionales sanitarios. Sin

\section{Referencias}

Anselm, A. H., Palda, V., Guest, C.B., McLean, R.F., Vachon, M.L.S., Kelner, M. y Lam-McCulloch, J. (2005). Barriers to communication regarding end-of-life care: perspectives of care providers. Journal of Critical Care, 20, 214-223. embargo, los pacientes vienen derivados tarde y sin conocer su estado de salud, lo que hace que se requiera mucho tiempo para poder trabajar su proceso (García-Caro et al., 2008a; Montoya-Juárez, 2006).

En la intervención con los familiares, lo que los psicólogos consideran más problemático coincide con lo destacado por otros estudios en equipos de cuidados paliativos: la existencia de psicopatología previa en los familiares y el paciente (Lichtenthal y Kissane, 2008), las grandes demandas de las familias (Anselm et al., 2005; Lichtenthal y Kissane, 2008), o la llamada obsesión terapéutica, que tiene que ver con la dificultad para suspender las medidas de carácter curativo (Mialdea et al., 2009). Durante las entrevistas se ha detectado en algunos psicólogos una cierta tolerancia hacia la llamada conspiración de silencio (Schmidt Río-Valle et al., 2009; Schmidt Río-Valle, Montoya-Juárez, García-Caro, y CruzQuintana, 2008). Ciertas actitudes, como intervenir a veces en primer lugar con la familia, para ver sobre qué puede hablarse con el paciente, o conocer qué es lo que sabe el paciente sobre su enfermedad, en lugar de preguntar directamente a él, parecen ser indicadores de ello (Schmidt RíoValle et al., 2009). Así, el psicólogo también puede pasar a formar parte, en ciertos momentos, de dicha conspiración. Sin embargo, también hay casos en los que el psicólogo se encarga de confrontar a las familias, para intentar hacer que dicho pacto de silencio se rompa.

El equipo de trabajo puede actuar como un factor de apoyo en las situaciones de fin de vida, o como un obstáculo, dependiendo del grado de integración del psicólogo, así como del ambiente que se respire en el mismo. Uno de los obstáculos con el que se encuentran los miembros de los equipos multidisciplinares que trabajan en el fin de vida es la falta de otros profesionales como ellos (Barbero y Díaz, 2007), el escaso tiempo disponible, así como la dificultad por parte del personal médico para delegarles ciertas intervenciones. Otros autores han encontrado resultados similares estudiando a equipos de cuidados paliativos (Goldsmith, Wittenberg-Lyles, Rodriguez, y Sanchez-Reilly, 2010), destacando además el desconocimiento por parte de los médicos, de los roles y funciones de los otros miembros del equipo multidisciplinar, y mostrando que el personal médico afirma sentir una mayor satisfacción laboral (Casarett, Spence, Haskins, y Teno, 2011).

Se puede concluir que existen una serie de obstáculos tanto a nivel emocional, como organizativo que influyen en la atención que prestan los psicólogos en el final de la vida. Se requieren, también, medidas de carácter formativo, así como una mejor distribución de los recursos para poder garantizar una intervención adecuada.
Barbero, J. y Díaz, L. (2007). Diez cuestiones inquietantes en cuidados paliativos. Anales del Sistema Sanitario de Navarra, 30, 71-89.

Barreto, P. y Bayés, R. (1990). El psicólogo ante el enfermo en situación terminal. Anales de Psicología, 6, 169-180. 
Bayés, R. (2001). Psicología del sufrimiento y de la muerte. Barcelona: Ediciones Martínez Roca.

Casado-Moragón, Á., Muñoz-Durán, G., Ortega-González, N. y Castellanos-Asenjo, A. (2005). Burnout en profesionales de los servicios paliativo, intensivo y urgencias de un gran hospital. Medicina Clínica, 124, 554-555.

Casarett, D. J., Spence, C., Haskins, M. y Teno, J. (2011). One big happy family? Interdisciplinary variation in job satisfaction among hospice providers. Journal of Palliative Medicine, 14, 913-917.

Fernández Fernández, M. E. (2003). Formación en Cuidados Paliativos por y para el psicólogo. Medicina Paliativa, 10, 39-46.

Flick, U. (2004). Introducción a la investigación cualitativa. Madrid: Morata.

García-Caro, M. P., Cruz-Quintana, F., Schmidt Río-Valle, J., MuñozVinuesa, A., Montoya-Juárez, R., Prados-Peña, D.,... Botella-López, M.C. (2010). Influencia de las emociones en el juicio clínico de los profesionales de la salud a propósito del diagnóstico de enfermedad terminal. International Journal of Clinical and Health Psychology, 10, 57-73.

García-Caro, M. P., Schmidt Río-Valle, J., Cruz-Quintana, F., Prados-Peña, D., Muñoz-Vinuesa, A. y Pappous, A. (2008a). Enfermedad terminal, muerte...en palabras de los profesionales (I). ROL de Enfermería, 31, $297-$ 302.

García-Caro, M. P., Schmidt Río-Valle, J., Cruz-Quintana, F., Prados-Peña, D., Muñoz-Vinuesa, A. y Pappous, A. (2008b). Enfermedad terminal, muerte...en palabras de los profesionales (y II). ROL de Enfermería, 31, 374-378.

García-Fernández, P., Arzate-Díaz, J., Bailón-Martínez, C., MartínezContreras, L.B., Torres-González, T., Castro-Martínez, M.G.,...PadillaNeri, H. (2008). Psicología de enlace en medicina interna. Medicina Interna de México, 24, 186-192.

Gardiner, C., Cobb, M., Gott, M. e Ingleton, C. (2011). Barriers to providing palliative care for older people in acute hospitals. Age and Ageing, 40, 233-238.

Glaser, B.G. y Strauss, A.L. (1967). The discovery of Grounded Theory: Strategies for qualitative research. Nueva York: Aldine

Goldsmith, J., Wittenberg-Lyles, E., Rodriguez, D. y Sanchez-Reilly, S. (2010). Interdisciplinary geriatric and palliative care team narratives: collaboration practices and barriers. Qualitative Health Research, 20, 93104.

Groot, M. M., Vernooij-Dassen, M., Crul, B. y Grol, R. (2005). General practitioners (GPs) and palliative care: perceived task and barriers in daily practice. Palliative Medicine, 19, 111-118.

Haley, W., Larson, D. G., Kasl-Godley, J., Neimeyer, R. A. y Kwilosz, D. M. (2003). Roles for psychologist in End-of-Life Care: Emerging models of practice. Professional Psychology: Research and Practice, 34, 626-633.

Lacasta, M. A., Rocafort-Gil, J., Blanco-Toro, L., Limonero-García, J. T. y Gómez Batiste, X. (2008). Intervención psicológica en Cuidados
Paliativos. Análisis de los servicios prestados en España. Medicina Paliativa, 15, 39-44.

Lewis, B. (2004) NVivo 2.0 and Atlas.ti 5.0: A Comparative Review of Two Popular Qualitative Data-Analysis Programs, Field Methods 16, 439-469

Lichtenthal, W. y Kissane, D. W. (2008). The management of family conflict in palliative care. Progress in Palliative Care, 16, 39-45.

López-Ríos, F. y Ortega-Ruiz, C. (2005). Intervención psicológica en cuidados paliativos: revisión y perspectivas. Clínica y Salud, 16, 143-160.

Mialdea, M. J., Sanz, J. y Sanz, A. (2009). Situaciones difíciles para el profesional de atención primaria en el cuidado al enfermo terminal. Psicooncología, 6, 53-63.

Montoya-Juárez, R. (2006). Aquellos que nos verán morir. Significado y respuesta de los profesionales sanitarios de una residencia de ancianos ante la muerte y los moribundos. Index de Enfermería, 52-53.

Muhr, T. (1991) Atlas-ti - A protoype for the support of text interpretation. Qualitative Sociology, 14, 349-371

Ostgathe, C. y Voltz, R. (2010). Quality indicators in end-of-life care. Current Opinion in Supportive and Palliative Care, 4, 170-173.

Pastrana, T., Jünger, S., Ostgathe, C., Elsner, F. y Radbruch, L. (2008). A matter of definition - key elements identified in a discourse analysis of definitions of palliative care. Palliative Medicine, 22, 222-232.

Pierce, B., Dougherty, E., Panzarella, T., Le, L., Rodin, G. y Zimmermann, C. (2007). Staff stress, work satisfaction, and death attitudes on an oncology palliative care unit, and on a medical and radiation oncology inpatient unit. Journal of Palliative Care, 23, 32-42.

Romaní-Costa, V., Expósito-López, A., Rodriguez-Carballeira, M. y Almagro-Mena, P. (2010). Morir en un hospital de agudos. Análisis de la situación de últimos días. Medicina Paliativa, 17, 222-225.

Romero, C., Álvarez, M., Bayés, R. y Schröder, M. (2001). ¿Cuándo se pide la intervención del psicólogo en una unidad de Cuidados Paliativos? Medicina Paliativa, 8, 170-172.

Schmidt Río-Valle, J., García-Caro, M. P., Montoya-Juárez, R., Prados-Peña D., Pappous, A. y Cruz-Quintana, F. (2009). Bad news for the patient and the family? The worst thing for the health care professional. Journal of Palliative Care, 25, 191-196.

Schmidt Río-Valle, J., Montoya-Juárez, R., García-Caro, M. P. y CruzQuintana, F. (2008). Dos procesos de fin de vida. Cuando la intervención de los profesionales marca la diferencia. Index de Enfermería, 17, 256-260.

Scientific Software Development (2011) ATLAS.ti Scientific Software Consultado el 15 de Septiembre de 2011 de, http://www.atlasti.com/es/index.html

Strauss, A. y Corbin, J. (1998) Basics of qualitative research: techniques and procedures for developing grounded theory. London: SAGE Publications

Werth Jr, J. L., Gordon, J. R. y Johnson Jr, R. (2002). Psychosocial issues near the end of life. Aging y Mental Health, 6, 402-412.

\section{Anexo: Estructura del Guión de la Entrevista}

$1^{a}$ Sección: Datos Sociodemográficos y Personales

$2^{a}$ Sección: Práctica Diaria y Sentimientos asociados

2.1 Descripción funcional del puesto de trabajo

2.2. Emociones y sentimientos que aporta el trabajo

$3^{\text {a }}$ Sección: Dificultades y experiencias en la intervención de los pacientes

3.1 Acompañamiento de los pacientes

3.2. Tipo de pacientes

3.3. Forma de intervenir

3.4. Obstáculos durante la intervención

$4^{\text {a }}$ Sección: Dificultades y experiencias en la intervención con las familias

4.1. Acompañamiento de las familias
4.2. Tipo de familias

4.3. Forma de intervenir

4.4. Obstáculos durante la intervención

$5^{a}$ Sección: Dificultades y experiencias con el equipo de trabajo

5.1. Lugar dentro del equipo

5.2. Relaciones con los compañeros

5.3. Obstáculos durante la intervención

$6^{a}$ Sección: Necesidades personales del psicólogo, avances y formación

6.1. Sentimientos y Efectos de la muerte

6.2. Avances en Cuidados Paliativos y el proceso de fin de vida

6.3. Necesidades formativas 